\title{
A theoretical insight in interactions of some chemical compounds as mTOR inhibitors
}

\author{
David Ebuka Arthur ${ }^{1 *}$, Jibrin Noah Akoji ${ }^{1}$, Riadh Sahnoun ${ }^{1}$, Greatman C. Okafor ${ }^{1}$, Karimatu Lami Abdullahi ${ }^{1}$, \\ Samira A. Abdullahi ${ }^{1}$ and Charles Mgbemena ${ }^{2}$
}

\begin{abstract}
Background: A series of known Food and Drug Administration (FDA) approved anticancer drugs were collected from the literature and docked against mTOR receptor which has been identified in present time as a target for therapeutic anticancer agents. The compounds binding affinity were calculated after minimising the interaction within the binding pockets' of the mTOR (4JT6) receptor.

Results: The result shows that PF-04691502 ligand best inhibited mTOR while occupying the Adenosine triphosphate (ATP)-binding site on the receptor. PF-04691502 had the best binding affinity with a reported value of - $39.261 \mathrm{kcal} / \mathrm{mol}$, and a hydrogen bond energy contribution of $-8.326 \mathrm{kcal} / \mathrm{mol}$. Polamid529 is also found to have a good binding affinity of $-36.75 \mathrm{kcal} / \mathrm{mol}$ with the receptor, but was less significant than that calculated for the reference or standard inhibitor (X6K) used $(-37.862 \mathrm{kcal} / \mathrm{mol}$ ). Further analysis revealed that Palomid529 formed a more stable complex with the receptor than torin 2 and X6K due to the significant hydrogen bond contributions it adds to its overall binding score.
\end{abstract}

Conclusion: PF-04691502 ligand was identified as the best inhibitor due to its high binding affinity for mTOR and should be considered as the best alternative to the reference inhibitor X6K.

Keywords: SBDD, Molecular docking, Binding energy, Hydrophobic interaction, Hydrogen interaction, Drug

\section{Background}

The mTOR kinase pathway is used for controlling growth of cells in the presence of nutrients, receptor growth factors and other external factors such as the environment, thereby causing cancer (Shaw and Cantley 2006; Zoncu et al. 2011; Laplante and Sabatini 2012; Chen and Long 2018; Paquette et al. 2018). The mTOR protein is an important growth receptor kinase, which is found in the phosphoinositide 3-kinase (PI3K)-related protein kinase (PIKK) family (Keith and Schreiber 1995).

mTOR is seen within two different complexes with inputs and resultant feedbacks. The first mTOR complex (mTORC1) is defined by its subunit Regulatory-associated protein of mTOR (RAPTOR) (Hara et al. 2002;

\footnotetext{
*Correspondence: davidebukaarthur@gmail.com

${ }^{1}$ Department of Chemistry, Baze University, Abuja, Nigeria

Full list of author information is available at the end of the article
}

Kim et al. 2002; Loewith et al. 2002), while in the second (mTORC2) is identified by Rapamycin-insensitive companion of mammalian target of mTOR (RICTOR) (Sarbassov et al. 2004). Both complexes contains the primary mLST8 subunit (Aylett et al. 2016), even though their number of subunits that forms chemical interactions with RAPTOR or RICTOR varies (Davis et al. 2014; Chen and Long 2018; Wu et al. 2018).

mTORC1 has been cited by so many authors to be responsible for cell growth through translation, biogenesis and autophagy (Matsuoka and Yashiro 2014; Meng et al. 2018; Paquette et al. 2018). On a second note, mTORC2 is responsible for promoting cell cycle entry, cell survival and anabolic output (Yang et al. 2013; Sciarretta et al. 2018; Wu et al. 2018).

Rapamycin, a known allosteric inhibitor forms a ternary complex with the FK506-binding protein 12 (FKBP12) and the FRB domain of mTOR (Choo and 
Blenis 2009). Rapamycin-FKBP12 inhibits mTORC $_{1}$ to an uneven scale that is dependent on phosphorylationsite (Choo and Blenis 2009), but it does not form complex with mTORC $_{2}$ (Bernard et al. 2020; Calejman et al. 2020; Knudsen et al. 2020). To overcome these limitations, ATP-competitive inhibitors that potently and evenly inhibit both mTORC $_{1}$ and mTORC $_{2}$ are now a driving focus in development of present day anticancer agents (Wander et al. 2011; Dienstmann et al. 2014; Jhanwar-Uniyal et al. 2019; Gericke et al. 2020).

Torin 2 specifically inhibits mTORDN-mLST8, and was reported to act by occupying the ATP site (Liu et al. 2011; Choi et al. 2020; Gericke et al. 2020). The molecules also shows that Torin2 trifluoromethyl group occupies the binding N-lobe pocket to reveal multiple determinants of mTOR specificity, including a deep conformational change within the catalytic cleft.
Here we present the molecular docking study results of mTOR receptor bound to several ATP-competitive inhibitors.

\section{Methods}

\section{Chemical dataset sources}

In this study, a chemical dataset of thirteen (13) compounds of known cancer drugs were collected from FDA library of approved drugs (www.bindingDB.org). The compounds were optimized at the density functional theory (DFT) level using Becks's three-parameter Lee-Yang-parr hybrid functional $\left(\mathrm{B}_{3} \mathrm{LYP}\right)$ at 6-31G* basis set (Davidson and Feller 1986; Schäfer et al. 1992) to simulate chemical structures of the molecules at their minimum potential energy or most stable state in a real chemical system before subjecting them to the molecular docking process (Table 1).

Table 1 Name, CAS number and chemical structure of the drugs

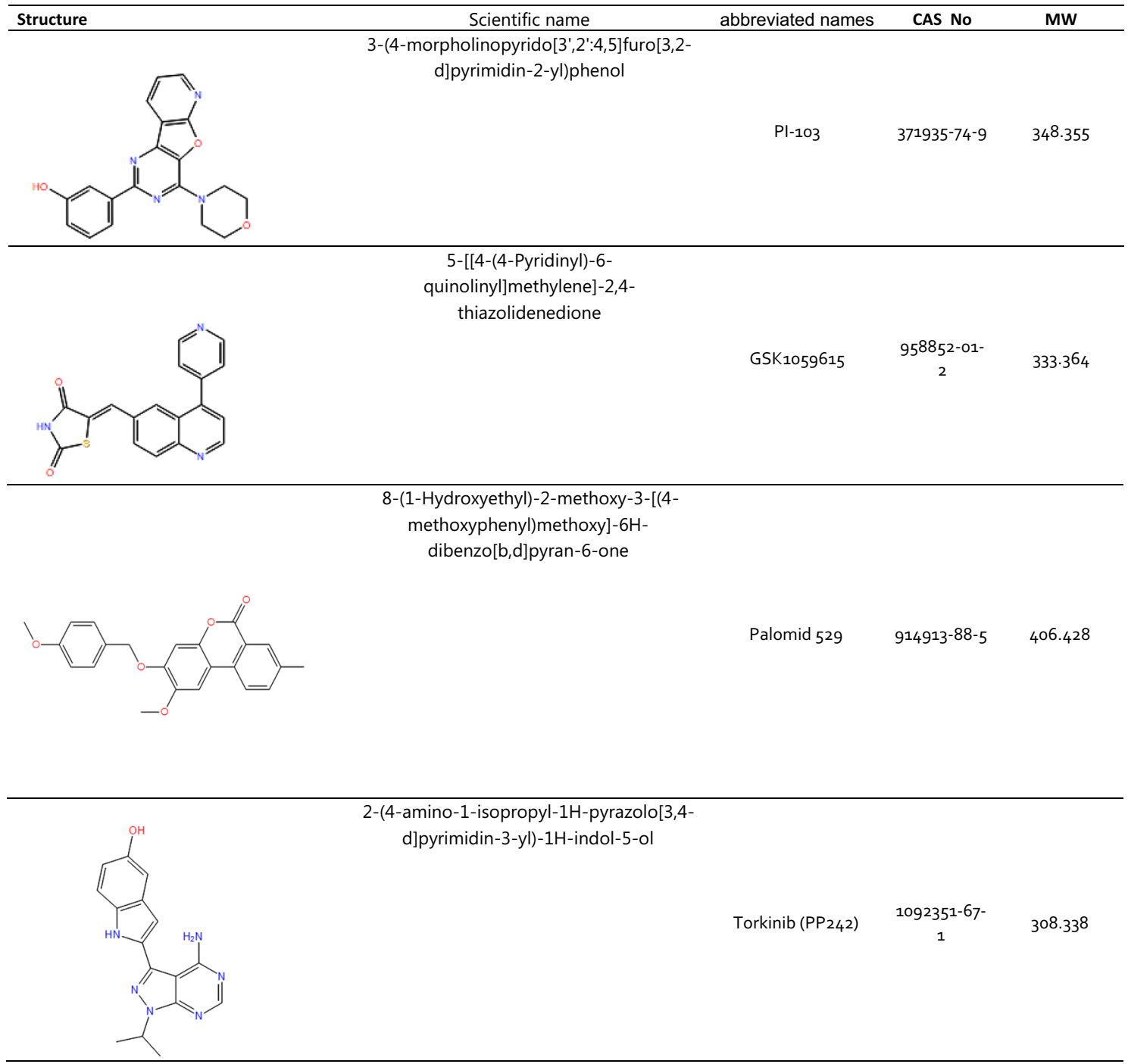


Table 1 (continued)

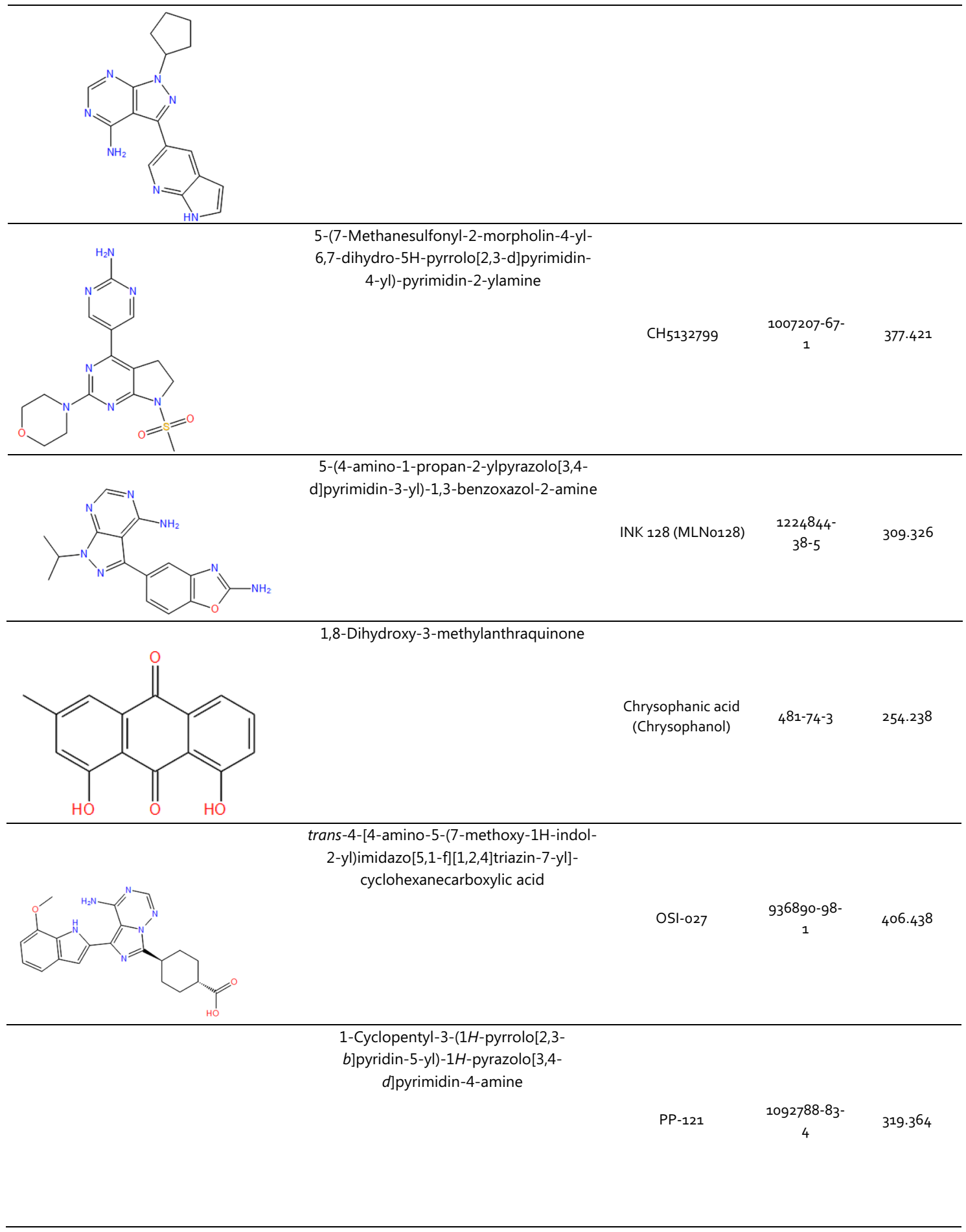

\section{Geometry optimization}

Optimization is the process of finding the equilibrium or concept energy geometry of molecules. Chemdraw software ultra-version 12.0 was used to draw the chemical structures of the compounds and subsequently imported into Spartan 14 software (Hehre and Huang 1995; Li, et al. 2004) to optimize the molecular geometry at the density functional theory (DFT) using $\mathrm{B}_{3} \mathrm{LYP}$ level of 
Table 1 (continued)

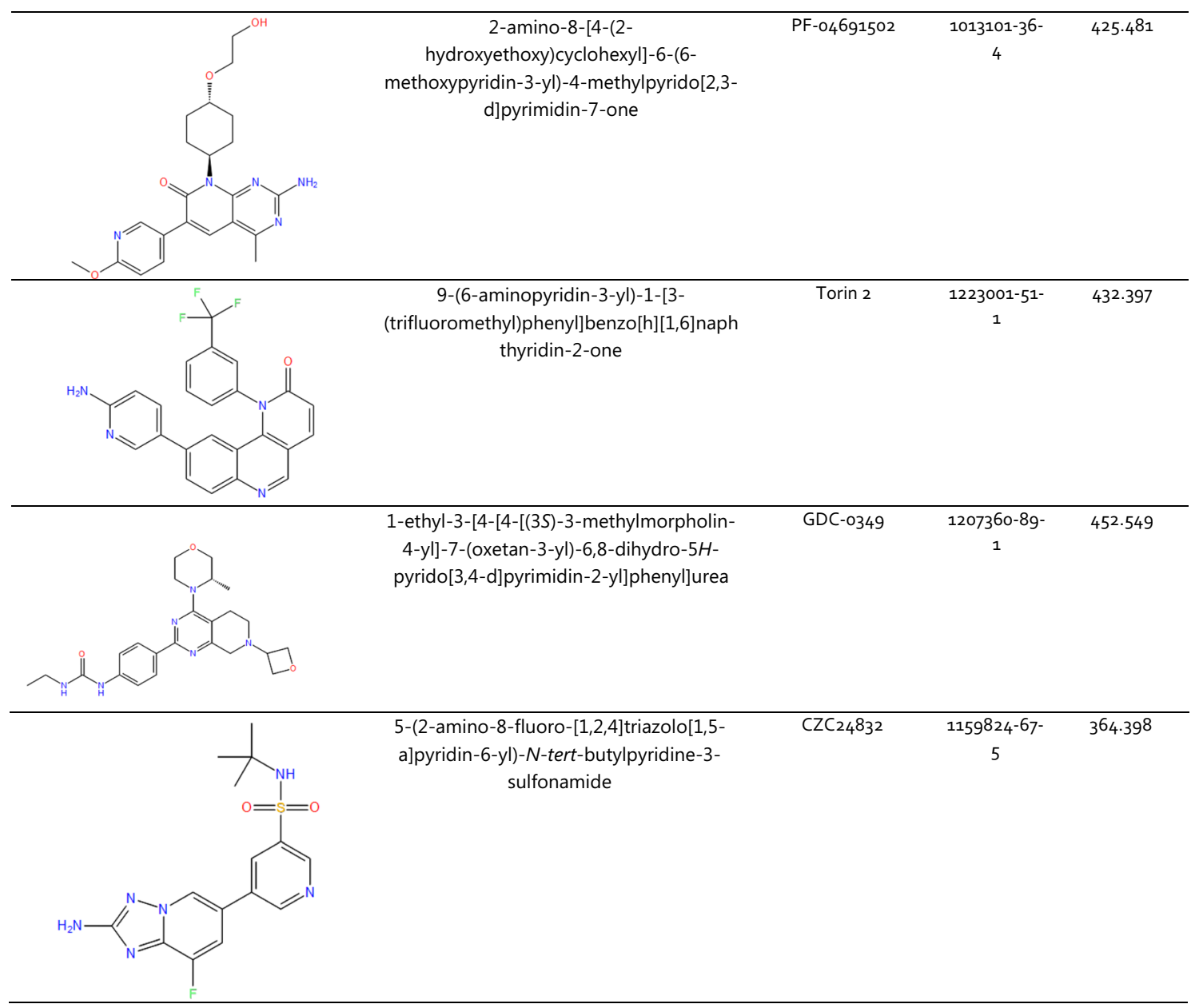

theory and 6-31G* as the basis set (Arthur and Abechi 2019; Arthur et al. 2019, 2020).

\section{Computational docking studies}

The molecular interactions studies were carried out on Dell computer system, with processor properties of Intel ${ }^{\circledR}$ Core i5-6100U CPU Dual@2.30 GHz, 12 GB (RAM) between the ligands and Mammalian target of rapamycin (mTOR) kinase (target); the X-ray Crystal structure of Mammalian target of rapamycin (mTOR) kinase, a transferase of MTOR deposited in RSCB protein data bank by Yang and his lab partners in 2013 (Yang et al. 2013).

\section{Preparation of ligand and target}

All the compounds were optimized using Spartan software initially saved as SDF files and were appropriately later saved as Protein Data Bank (PDB) file. Subsequently the PDB file of the receptor were downloaded from RSCB site (http://www.rcsb.org/) with the PDB ID: 4JT6 for the crystal structure of Mammalian target of rapamycin (mTOR) kinase. The X-ray diffraction resolution for 4JT6 was given as 3.60 amstrong unit (Yang et al. 2013). Figure 1 displays the prepared structure of the receptor.

\section{Detailed docking process on internal coordinate mechanics (ICM) molsoft program}

The process involves setting up the project file by reading in the PDB file of the receptor. For example, the PDB file for WT protective antigen imported into ICM pro. The PDB file was converted into an ICM object by deleting all water molecules present in the binding pocket and selecting the option to optimise all hydrogens and optimise HisProAsnGlnCys. The box hide missing side chains was unchecked. Since the ICM is required to build missing heavy atoms that are not reported in the PDB (due to the lack of density), they are added according to the residue name and assigned zero occupancies. The next step involves moving any ligand that might be complexed with 


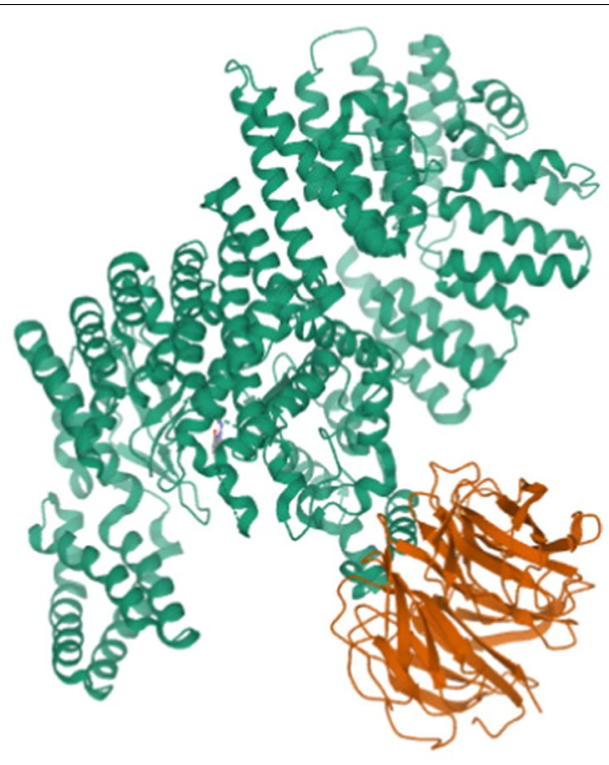

PDB ID: 4JT6 (3.60Å)

Fig. 1 Prepared structures of the target

the receptor out of the pocket of mTOR kinase (4JT6), otherwise the ligand will be included in the docking maps which will ultimately affect the result (Arthur and Uzairu 2018; Arthur et al. 2019). The ligand was removed from the ICM object by right clicking on the ligand in the ICM Workspace and then selecting "move from object".

Afterwards we generated the receptor grid binding site map. The box was made large enough to encompass the binding pocket but not too large to include regions of the receptor which are not relevant for the ligand to bind. Once

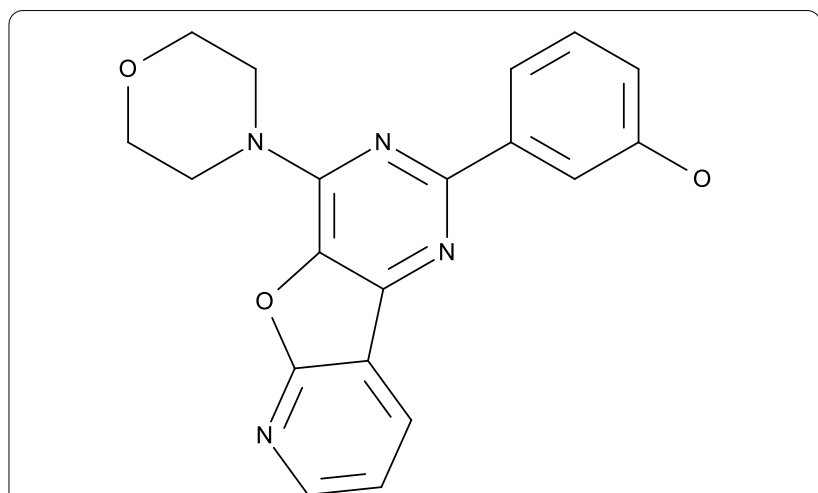

Fig. 2 2D structure of the reference ligand 3-(4-morpholin-4-ylpyr ido[3',2':4,5]furo[3,2-d]pyrimidin-2-yl)phenol (X6K). 2D 2dimension

the receptor and maps have been correctly set up, then the docking procedure could begin. The SD files of the optimised ligands are imported into the ICM programme as chemical spreadsheet. To dock directly from a chemical table, the chemicals in the table to be docked were selected. On the docking menu, dock chemical table was selected and after the docking process is finished, the hitlist were displayed. The docking results are then exported as a spreadsheet file saved in csv format. After docking the binding modes and interaction of the best ligand-receptor complexes are displayed by right clicking the ligand docked in the ICM window and saving the file output as a pdb file. The saved file was then imported into the discovery studio visualiser, where the 2D and $3 \mathrm{D}$ interactions could be properly seen.

The receptors, after treatment, were docked with their respective ligands. Five types of interaction potentials that represent the receptor pocket includes van der Waals

Table 2 Name, binging score and contributions of interaction energies to the binding affinity

\begin{tabular}{|c|c|c|c|c|c|c|c|c|c|c|}
\hline Name & CAS No & Score (kcal/mol /mol) & Nflex & Hbond & Hphob & Vwlnt & Eintl & Dsolv & SolEl & mfScore \\
\hline X6K & & -37.862 & 0 & -5.113 & -7.187 & -31.281 & 3.142 & 12.023 & 3.284 & -165.733 \\
\hline $\mathrm{Pl}-103$ & $371935-74-9$ & -34.361 & 0 & -4.677 & -7.408 & -30.102 & 2.951 & 12.542 & 4.743 & -159.424 \\
\hline GSK1059615 & $958852-01-2$ & -33.574 & 0 & -5.670 & -5.914 & -30.258 & 8.390 & 12.201 & 8.850 & -132.520 \\
\hline Palomid 529 & 914913-88-5 & -36.751 & 4 & -6.412 & -8.618 & -32.604 & 9.630 & 14.586 & 6.835 & -110.552 \\
\hline Torkinib (PP242) & 1092351-67-1 & -31.330 & 1 & -7.059 & -6.368 & -28.849 & 4.308 & 16.633 & 9.953 & -135.032 \\
\hline $\begin{array}{l}\text { Chrysophanic acid } \\
\text { (Chrysophanol) }\end{array}$ & $481-74-3$ & -34.447 & 0 & -6.098 & -4.899 & -28.956 & 0.617 & 8.957 & 9.851 & -96.226 \\
\hline OSI-027 & 936890-98-1 & -35.121 & 3 & -6.732 & -7.266 & -33.715 & 8.355 & 18.261 & 7.572 & -151.135 \\
\hline PP-121 & 1092788-83-4 & -33.602 & 1 & -7.297 & -6.372 & -27.813 & 6.796 & 14.258 & 8.559 & -141.058 \\
\hline CH5132799 & 1007207-67-1 & -31.286 & 1 & -4.110 & -7.006 & -31.648 & 5.898 & 13.459 & 7.212 & -150.136 \\
\hline INK 128 (MLN0128) & $1224844-38-5$ & -30.828 & 1 & -7.551 & -5.755 & -26.544 & 6.389 & 17.407 & 7.608 & -125.611 \\
\hline PF-04691502 & $1013101-36-4$ & -39.261 & 5 & -8.326 & -8.325 & -36.377 & 16.339 & 18.901 & 9.972 & -156.649 \\
\hline Torin 2 & $1223001-51-1$ & -34.794 & 1 & -2.910 & -7.662 & -36.370 & 7.496 & 10.956 & 7.964 & -179.998 \\
\hline GDC-0349 & $1207360-89-1$ & -32.260 & 4 & -4.017 & -9.754 & -38.017 & 6.122 & 16.924 & 11.156 & -135.825 \\
\hline CZC24832 & $1159824-67-5$ & -25.350 & 3 & -3.811 & -5.184 & -30.471 & 2.904 & 14.622 & 8.287 & -104.365 \\
\hline
\end{tabular}

Bold values indicate the location of the most active ligand under study 


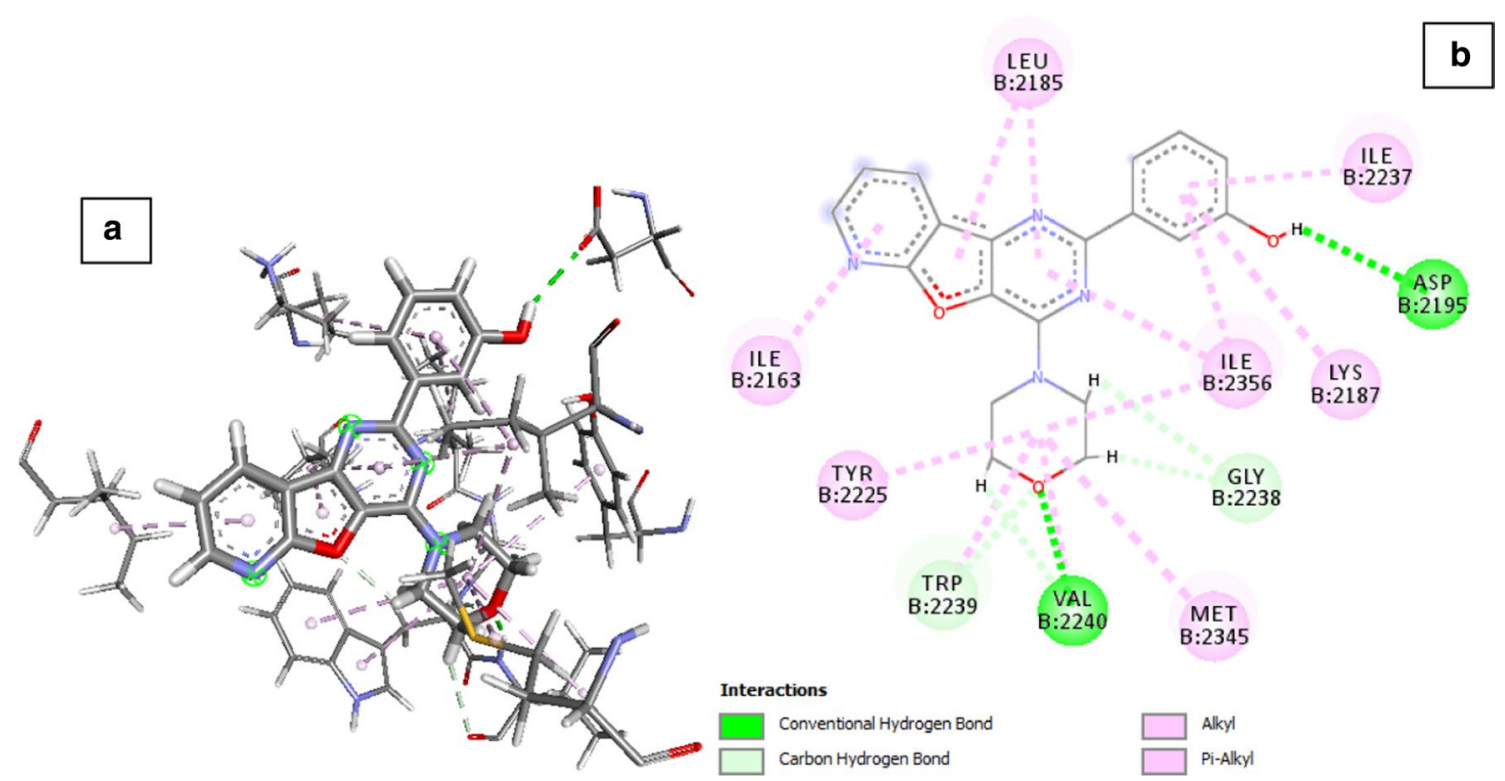

Fig. $33 \mathrm{D}$ and 2D binding pose interaction of X6K-mTOR kinase complex in $\mathbf{a}$ and $\mathbf{b}$ respectively. 2D 2dimension, 3D 3dimension

Table 3 Types of interactions in the Reference ligand (X6K)-4JT6 (mTOR) receptor complex

\begin{tabular}{|c|c|c|c|c|c|c|c|}
\hline Distance & Types & From & From Chemistry & To & To Chemistry & Angle DHA & Angle HAY \\
\hline 1.766 & Conventional Hydrogen Bond & B:VAL2240:HN & H-Donor & :RES1:02 & H-Acceptor & 151.351 & 123.36 \\
\hline 2.275 & Conventional Hydrogen Bond & :RES1:H01 & H-Donor & B:ASP2195:OD2 & H-Acceptor & 153.335 & 136.905 \\
\hline 2.653 & Carbon Hydrogen Bond & B:TRP2239:HA & H-Donor & $: \mathrm{RES1}: \mathrm{O} 2$ & H-Acceptor & 138.065 & 105.269 \\
\hline 2.762 & Carbon Hydrogen Bond & :RES1:H101 & H-Donor & B:GLY2238:O & H-Acceptor & 112.187 & 161.952 \\
\hline 2.775 & Carbon Hydrogen Bond & :RES1:H111 & H-Donor & B:VAL2240:O & H-Acceptor & 146.604 & 124.89 \\
\hline 2.254 & Carbon Hydrogen Bond & :RES1:H121 & H-Donor & :RES1:O1 & H-Acceptor & 116.681 & 96.896 \\
\hline 2.715 & Carbon Hydrogen Bond & :RES1:H91 & H-Donor & B:GLY2238:O & H-Acceptor & 125.859 & 133.916 \\
\hline 5.115 & Alkyl & B:VAL2240 & Alkyl & :RES1 & Alkyl & & \\
\hline 5.185 & Alkyl & B:MET2345 & Alkyl & :RES1 & Alkyl & & \\
\hline 5.368 & Alkyl & B:ILE2356 & Alkyl & :RES1 & Alkyl & & \\
\hline 5.496 & Pi-Alkyl & B:TYR2225 & Pi-Orbitals & :RES1 & Alkyl & & \\
\hline 4.846 & Pi-Alkyl & B:TRP2239 & Pi-Orbitals & :RES1 & Alkyl & & \\
\hline 5.281 & Pi-Alkyl & B:TRP2239 & Pi-Orbitals & :RES1 & Alkyl & & \\
\hline 4.880 & Pi-Alkyl & :RES1 & Pi-Orbitals & B:ILE2163 & Alkyl & & \\
\hline 4.864 & Pi-Alkyl & :RES1 & Pi-Orbitals & B:LEU2185 & Alkyl & & \\
\hline 4.640 & Pi-Alkyl & :RES1 & Pi-Orbitals & B:LEU2185 & Alkyl & & \\
\hline 4.398 & Pi-Alkyl & :RES1 & Pi-Orbitals & B:ILE2356 & Alkyl & & \\
\hline 5.402 & Pi-Alkyl & :RES1 & Pi-Orbitals & B:LYS2187 & Alkyl & & \\
\hline 4.650 & Pi-Alkyl & :RES1 & Pi-Orbitals & B:ILE2237 & Alkyl & & \\
\hline 4.956 & Pi-Alkyl & :RES1 & Pi-Orbitals & B:ILE2356 & Alkyl & & \\
\hline
\end{tabular}

potential for a hydrogen atom probe, van der Waals potential for a heavy-atom probe (generic carbon of $1.7 \AA$ radius), optimised electrostatic term, hydrophobic terms and lonepair-based potential which reflects directional preferences in hydrogen bonds calculated. These energy terms are based on the all-atom vacuum force field ECEPP/3 with added functions to account for solvation free energy and entropic contribution. The score was calculated from

$$
E_{\mathrm{bind}}=E_{\mathrm{int}}+T \Delta S_{\mathrm{Tor}}+E_{\mathrm{VW}}+\alpha_{1} E_{\mathrm{el}}+\alpha_{2} E_{\mathrm{hb}}+\alpha_{3} E_{\mathrm{hp}} \alpha_{4} E_{\mathrm{sf}}
$$




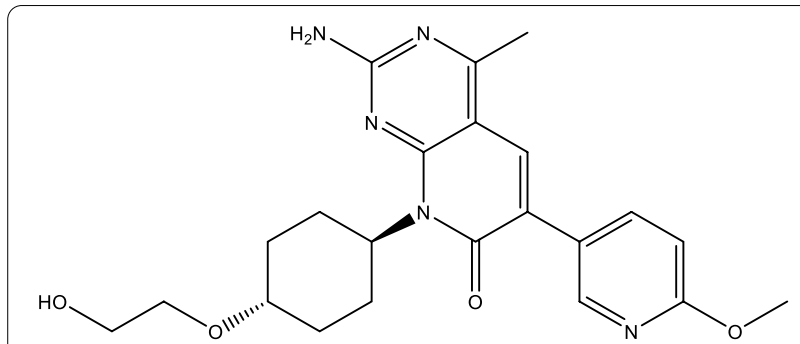

Fig. 4 2D structure of PF-04691502 ligand. 2D 2dimension

In Eq. (1), $E_{\mathrm{vw}}, E_{\mathrm{e}}, E_{\mathrm{hb}}, E_{\mathrm{hp}}$, and $E_{\mathrm{sf}}$ are van der Waals, electrostatic, hydrogen bonding, and non-polar and polar atom solvation energy differences between bound and unbound states, respectively. $E_{\text {int }}$ is the ligand internal strain, $\Delta S_{\text {Tor }}$ is its conformational entropy loss upon binding, $T=300 \mathrm{~K}$, and $\alpha i$ are ligand and receptor independent constants. Each compound was docked to the protein binding pocket, and a score from the docking was assigned to each compound according to the weighed component of the ICM scoring function. Each compound was docked ten times to ensure the convergence of the Monte Carlo optimisation, and the minimum score of each ligand from the ten independent docking experiments was retained and used for ranking (Neves et al. 2012).

The conformational sampling in the programme is based on the biased probability Monte Carlo (BPMC) procedure, which randomly selects a conformation in the internal coordinate space and then makes a step to a new random position independent of the previous one but according to a predefined continuous probability distribution. It has also been shown that after each random step, full local minimisation greatly improves the efficiency of the procedure (Arthur et al. 2018, 2019; Abdullahi et al. 2020). The ICM programme relies on global optimisation of the entire flexible ligand in the receptor field and combines large-scale random moves of several types with gradient local minimisation and a search history mechanism.

\section{Virtual screening}

The scoring function should give a good approximation of the binding free energy between a ligand and a receptor and is usually a function of different energy terms based on a force-field. The ICM scoring function is weighted according to the following parameters

1. internal force-field energy of the ligand,

2. entropy loss of the ligand between bound and unbound states,

3. ligand-receptor hydrogen bond interactions,

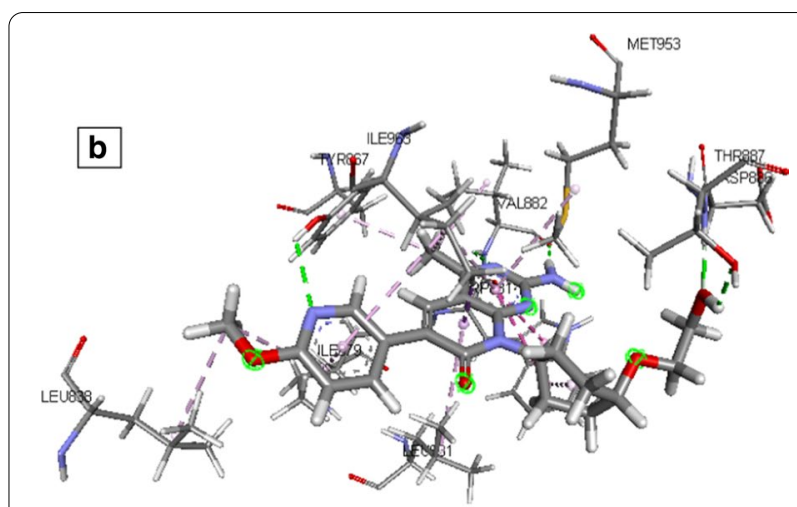

$\mathbf{a}$

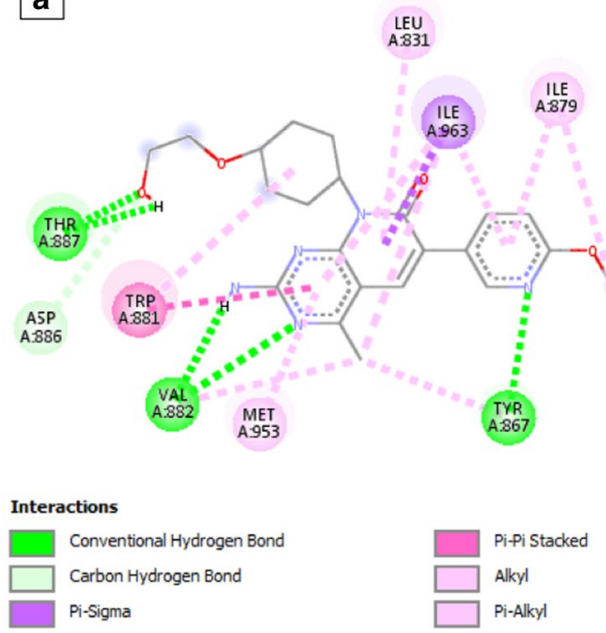

Fig. 5 3D and 2D binding pose interaction of PF-04691502-mTOR kinase complex in $\mathbf{b}$ and $\mathbf{a}$ respectively. $2 \mathrm{D} 2$ dimension, $3 \mathrm{D}$ 3dimension

4. polar and non-polar solvation energy differences between bound and unbound states,

5. electrostatic energy,

6. hydrophobic energy, and

7. hydrogen bond donor or acceptor desolvation.

The lower the ICM score, the higher the chance the ligand is a binder.

\section{Results}

The docking result of this study is presented in terms of binding affinity $(\mathrm{kcal} / \mathrm{mol} / \mathrm{mol})$ as reported in Table 2 along with their number of flexible bonds (Nflex), hydrogen bond energy (Hbond), hydrophobic bond energy (Hphob) values and the compound IDs as collected from BindingDB library. All the ligands were docked into the active site of the receptor (crystal structure of structure 
Table 4 Types of interactions in the PF-04691502-4JT6 (mTOR) receptor complex

\begin{tabular}{|c|c|c|c|c|c|c|c|}
\hline Distance & Types & From & From Chemistry & To & To Chemistry & Angle DHA & Angle HAY \\
\hline 1.769 & Conventional Hydrogen Bond & A:TYR867:HH & H-Donor & :RES1:O2 & H-Acceptor & 164.087 & 114.502 \\
\hline 1.897 & Conventional Hydrogen Bond & A:VAL882:HN & H-Donor & :RES1:03 & H-Acceptor & 151.228 & 123.806 \\
\hline 2.653 & Carbon Hydrogen Bond & A:TRP881:HA & H-Donor & :RES1:O3 & H-Acceptor & 141.934 & 104.673 \\
\hline 2.820 & Carbon Hydrogen Bond & :RES1:H11 & H-Donor & A:GLY880:O & H-Acceptor & 109.087 & 162.538 \\
\hline 2.234 & Carbon Hydrogen Bond & :RES1:H16 & H-Donor & :RES1:01 & H-Acceptor & 116.259 & 97.605 \\
\hline 2.713 & Carbon Hydrogen Bond & :RES1:H9 & H-Donor & A:GLY880:O & H-Acceptor & 127.216 & 138.529 \\
\hline 5.235 & Alkyl & A:VAL882 & Alkyl & :RES1 & Alkyl & & \\
\hline 5.295 & Alkyl & A:MET953 & Alkyl & :RES1 & Alkyl & & \\
\hline 5.170 & Alkyl & A:ILE963 & Alkyl & :RES1 & Alkyl & & \\
\hline 5.259 & Pi-Alkyl & A:TYR867 & Pi-Orbitals & :RES1 & Alkyl & & \\
\hline 5.096 & Pi-Alkyl & A:TRP881 & Pi-Orbitals & :RES1 & Alkyl & & \\
\hline 5.424 & Pi-Alkyl & A:TRP881 & Pi-Orbitals & :RES1 & Alkyl & & \\
\hline 4.764 & Pi-Alkyl & :RES1 & Pi-Orbitals & A:LEU831 & Alkyl & & \\
\hline 4.657 & Pi-Alkyl & :RES1 & Pi-Orbitals & A:LEU831 & Alkyl & & \\
\hline 5.300 & Pi-Alkyl & :RES1 & Pi-Orbitals & A:ILE879 & Alkyl & & \\
\hline 4.155 & Pi-Alkyl & :RES1 & Pi-Orbitals & A:ILE963 & Alkyl & & \\
\hline 5.212 & Pi-Alkyl & :RES1 & Pi-Orbitals & A:ILE963 & Alkyl & & \\
\hline 4.390 & Pi-Alkyl & :RES1 & Pi-Orbitals & A:ILE879 & Alkyl & & \\
\hline 5.432 & Pi-Alkyl & :RES1 & Pi-Orbitals & A:ILE963 & Alkyl & & \\
\hline 5.162 & Pi-Alkyl & :RES1 & Pi-Orbitals & A:ILE804 & Alkyl & & \\
\hline 5.367 & Pi-Alkyl & :RES1 & Pi-Orbitals & A:LEU831 & Alkyl & & \\
\hline
\end{tabular}

of mTORdeltaN) in order to evaluate their abilities to mTOR kinase.

X6K whose IUPAC name is 3-(4-morpholin4-ylpyrido $\left[3^{\prime}, 2^{\prime}: 4,5\right]$ furo[3,2-d]pyrimidin-2-yl)phenol was found in complex with the receptor 4JT6. It was removed from the receptor and later redocked as reference inhibitor to be used in comparison with all other studied compounds.

\section{Discussion}

The molecular docking result of the reference inhibitor is presented along with the docking scores of the chemical dataset in Table 2. The reference inhibitor (X6K) was reported to have a binding energy of $-37.862 \mathrm{kcal} / \mathrm{mol} /$ $\mathrm{mol}$, the score shows a very strong affinity for the active site and hence high inhibition efficiency of the inhibitor. The contributions from hydrogen bonds, hydrophobic

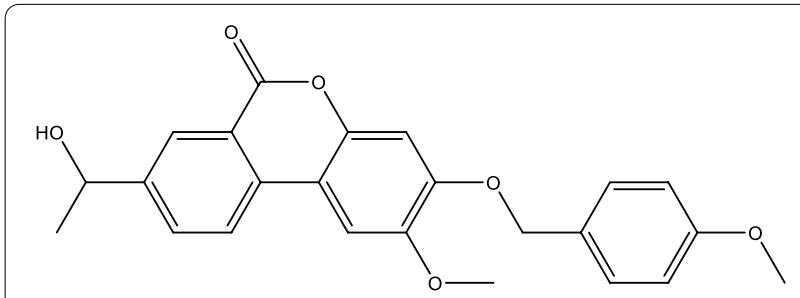

Fig. 6 2D structure of Palomid 529 ligand. 2D 2dimension interactions and van der Waals interactions were significant to the high affinity for the binding site of the receptor.

The binding scores of the other inhibitors confirm that they are all active and effectively binds with the active sites. The binding energy of PF-04691502 was the least, its score was significantly lower than all the other inhibitors and also that of the referenced drug. The binding score of PF-04691502 is reported in Table 2 as $-39.261 \mathrm{kcal} / \mathrm{mol}$, and from the table it is evident that the exceedingly low value was due to the high number of hydrogen bonds that is formed by the ligand leading to the significant value $(-8.326 \mathrm{kcal} / \mathrm{mol})$ of hydrogen bond energy contribution (Fig. 2).

The 2 and 3-dimensional pose of $\mathrm{X} 6 \mathrm{~K}$ in the active site of the mTOR kinase is presented in Fig. 3. The 2-dimensional pose shows clearly that two distinct conventional hydrogen bonds were formed between the phenol fragment of X6K and Aspartic acid (B:2195), while the other way between value (B:2240) and the morpholinyl oxygen of X6K with VAL. A more detail descriptors of the other interactions formed can be seen in Table 3.

The hydrogen bond formed by the phenol fragment had the shortest bond length of $1.766 \AA$, this shows that the bond fastens $\mathrm{X} 6 \mathrm{~K}$ to receptor active site. In this case X6K acts as $\mathrm{H}$-acceptor forming a DHA angle of 151.351 which are close to $180^{\circ}$ found in linear bonds. Table 3 


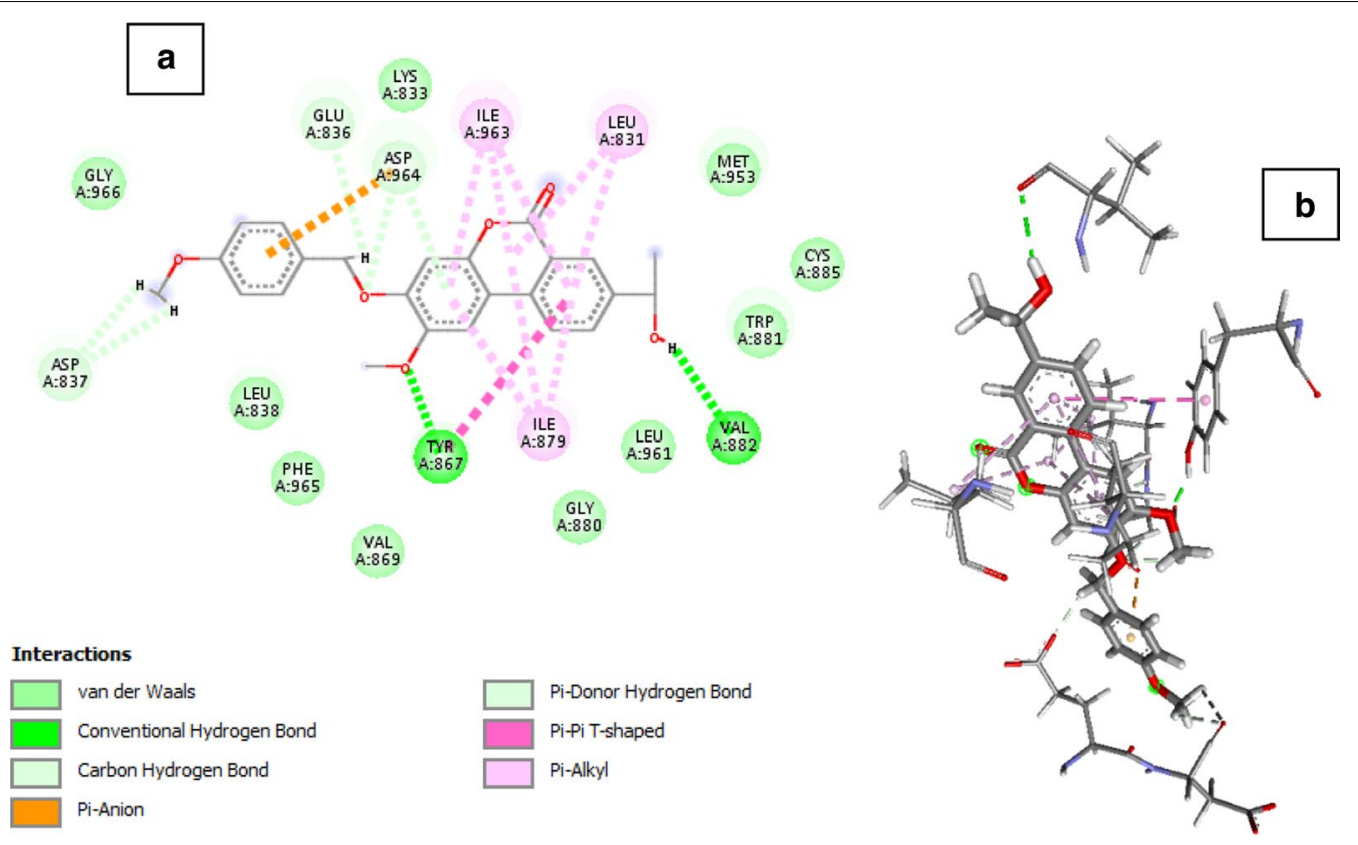

Fig. 7 2D and 3D binding pose interaction of Palomid 529-mTOR kinase complex in $\mathbf{a}$ and $\mathbf{b}$ respectively. 2D 2dimension, 3D 3dimension

Table 5 Types of interactions in the Palomid 529-4JT6 (mTOR) receptor complex

\begin{tabular}{|c|c|c|c|c|c|}
\hline Distance & Types & From & From Chemistry & To & To Chemistry \\
\hline 1.650 & Conventional Hydrogen Bond & A:TYR867:HH & H-Donor & :RES1:O3 & H-Acceptor \\
\hline 2.300 & Conventional Hydrogen Bond & :RES1:H22 & H-Donor & A:VAL882:O & H-Acceptor \\
\hline 2.487 & Carbon Hydrogen Bond & A:ASP964:HA & H-Donor & :RES1:O2 & H-Acceptor \\
\hline 2.301 & Carbon Hydrogen Bond & :RES1:H13 & H-Donor & :RES1:O2 & H-Acceptor \\
\hline 2.969 & Carbon Hydrogen Bond & :RES1:H5 & H-Donor & A:ASP837:O & H-Acceptor \\
\hline 2.514 & Carbon Hydrogen Bond & :RES1:H7 & H-Donor & A:ASP837:O & H-Acceptor \\
\hline 2.072 & Carbon Hydrogen Bond & :RES1:H9 & H-Donor & A:GLU836:OE1 & H-Acceptor \\
\hline 3.481 & Pi-Anion & A:ASP964:OD1 & Negative & :RES1 & Pi-Orbitals \\
\hline 2.927 & Pi-Donor Hydrogen Bond & A:ASP964:HN & H-Donor & :RES1 & Pi-Orbitals \\
\hline 5.382 & Pi-PiT-shaped & A:TYR867 & Pi-Orbitals & :RES1 & Pi-Orbitals \\
\hline 4.250 & Pi-Alkyl & :RES1 & Pi-Orbitals & A:ILE879 & Alkyl \\
\hline 4.362 & Pi-Alkyl & :RES1 & Pi-Orbitals & A:ILE963 & Alkyl \\
\hline 4.998 & Pi-Alkyl & :RES1 & Pi-Orbitals & A:LEU831 & Alkyl \\
\hline 4.904 & Pi-Alkyl & :RES1 & Pi-Orbitals & A:ILE879 & Alkyl \\
\hline 3.954 & Pi-Alkyl & :RES1 & Pi-Orbitals & A:ILE963 & Alkyl \\
\hline 5.354 & Pi-Alkyl & :RES1 & Pi-Orbitals & A:LEU831 & Alkyl \\
\hline 5.417 & Pi-Alkyl & :RES1 & Pi-Orbitals & A:ILE879 & Alkyl \\
\hline 4.342 & Pi-Alkyl & :RES1 & Pi-Orbitals & A:ILE963 & Alkyl \\
\hline
\end{tabular}

also shows that 3-alkyl-alkyl bond, and 10 pi-alkyl bonds were formed between the residue (X6K) and the receptor.

The 2D structure of PF-04691502 ligand is presented in Fig. 4, and was found to bond best with the mTORreceptor (Fig. 5) when compared to all the other molecules in Table 2.
The binding energy of PF-04691502 was reported as - $39.261 \mathrm{kcal} / \mathrm{mol}$ in Table 2 and it shows that PF inhibited mTOR receptor more than the referenced ligand and other molecules on the table. The significance of the binding affinity was found to be as a result of the very low 


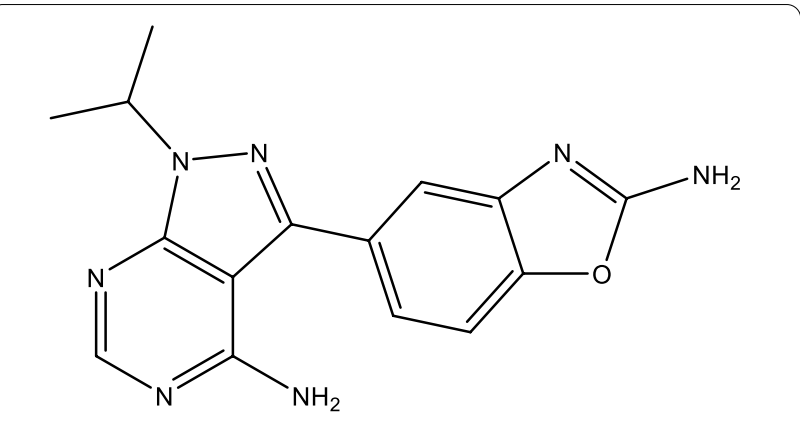

Fig. 8 2D structure of INK 128 (MLN0128) ligand. 2D 2dimension

hydrogen bond energy between PF and 4JT6 (mTOR) in Table 2 given as $-8.326 \mathrm{kcal} / \mathrm{mol}$.

The binding poses in figure show that the pyridolfragment was significant to the attachment of the ligand on the binding site of mTOR kinase receptor, it can be seen that the morpholinyl oxygen on pyrimidin backbone of the PF-04691502 land formed a hydrogen bond with VAL 882 which was firmly held by the bond distance reported in Table 4 as $1.897 \mathrm{~A}$, while the phenol group on the ligand stabilized the right-base corner of the complex by forming a strong hydrogen bond with TYR 867.

Other interactions that were noticeable within the complex formed includes carbon hydrogen bonds which were weak interactions, while the pi-alkyl and alkyl interactions present helped in stabilizing the conformation of the ligand as well as intercalating the structure with the binding pocket of the receptor.

Palomid 529 and INK 128 are two other ligands with high binding affinity for mTOR receptor binding pocket. The 2D structure of Palomid 529 in Fig. 6, shows that the chemical structure contains a lactone-ring and three alkoxy groups which formed important interactions with the surrounding amino acids in the binding pocket (Fig. 7).

Two hydrogen bonds were formed between Palomid 529 and 4JT6 (mTOR) receptor, the binding energy was reported as $-36.751 \mathrm{kcal} / \mathrm{mol}$ and can be seen to inhibit the receptor less when compared to PF and referenced ligand (X6K). All the interaction types involved in the Palomid 529-4JT6 (mTOR) receptor complex are presented in Table 5. The presence of pi-anion and pi-alkyl interaction helped in the intercalation of the ligand in the binding pocket of the receptor.

The 2D molecular structure of INK 128 presented in Fig. 8 shows that the structure contains a $1 \mathrm{H}$-pyrazolo[3,4-d]pyrimidine fragment on the left side, while on the right side a benzo[d]oxazol-2-amine fragment. The presence of the amine groups on these fragments were responsible for the significant number of hydrogen bonds formed by INK 128 in the binding pocket shown in Fig. 9.

From the Table 2, it was observed that INK 128 (MLN0128) had binding energy $(-30.828 \mathrm{kcal} / \mathrm{mol}$ ) higher than that of the referenced compound X6K.

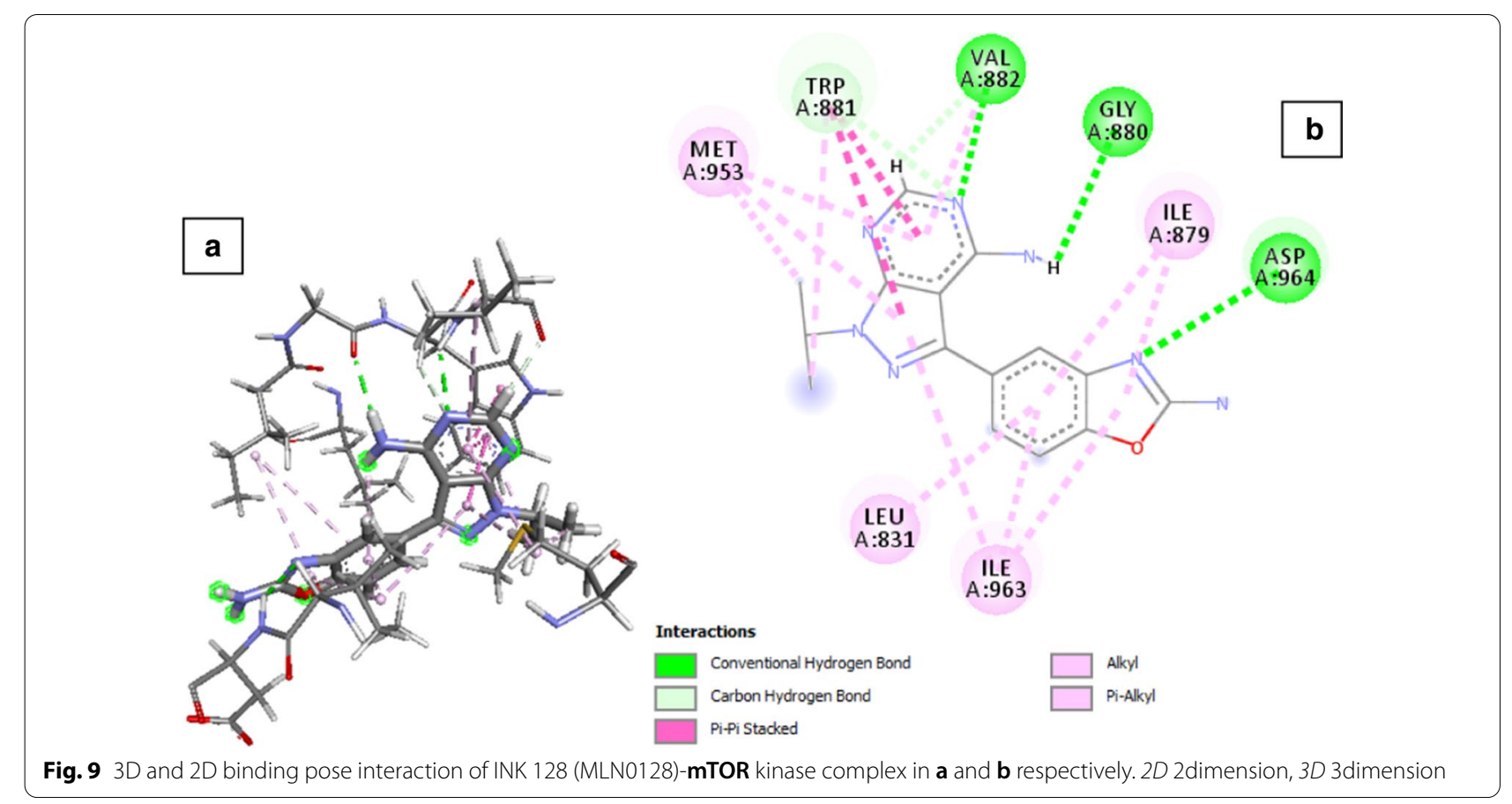


Table 6 Types of interactions in the INK-4JT6 (mTOR) receptor complex

\begin{tabular}{|c|c|c|c|c|c|}
\hline Distance & Types & From & From Chemistry & To & To Chemistry \\
\hline 2.175 & Conventional Hydrogen Bond & A:VAL882:HN & H-Donor & :RES1:N1 & H-Acceptor \\
\hline 2.825 & Conventional Hydrogen Bond & A:ASP964:HN & H-Donor & :RES1:N6 & H-Acceptor \\
\hline 2.111 & Conventional Hydrogen Bond & :RES1:H9 & H-Donor & A:GLY880:O & H-Acceptor \\
\hline 2.619 & Carbon Hydrogen Bond & A:TRP881:HA & H-Donor & :RES1:N1 & H-Acceptor \\
\hline 2.069 & Carbon Hydrogen Bond & :RES1:H1 & H-Donor & A:VAL882:O & H-Acceptor \\
\hline 3.977 & Pi-Pi Stacked & A:TRP881 & Pi-Orbitals & :RES1 & Pi-Orbitals \\
\hline 4.787 & Pi-Pi Stacked & A:TRP881 & Pi-Orbitals & :RES1 & Pi-Orbitals \\
\hline 4.372 & Pi-Pi Stacked & A:TRP881 & Pi-Orbitals & :RES1 & Pi-Orbitals \\
\hline 4.177 & Pi-Pi Stacked & :RES1 & Pi-Orbitals & A:TRP881 & Pi-Orbitals \\
\hline 4.107 & Alkyl & :RES1:C7 & Alkyl & A:MET953 & Alkyl \\
\hline 4.767 & Pi-Alkyl & A:ILE879 & Pi-Orbitals & :RES1 & Alkyl \\
\hline 4.430 & Pi-Alkyl & A:TRP881 & Pi-Orbitals & :RES1:C8 & Alkyl \\
\hline 4.485 & Pi-Alkyl & A:ILE963 & Pi-Orbitals & :RES1 & Alkyl \\
\hline 5.357 & Pi-Alkyl & :RES1 & Pi-Orbitals & A:VAL882 & Alkyl \\
\hline 4.823 & Pi-Alkyl & :RES1 & Pi-Orbitals & A:MET953 & Alkyl \\
\hline 4.810 & Pi-Alkyl & :RES1 & Pi-Orbitals & A:LEU831 & Alkyl \\
\hline 5.451 & Pi-Alkyl & :RES1 & Pi-Orbitals & A:ILE879 & Alkyl \\
\hline 4.271 & Pi-Alkyl & :RES1 & Pi-Orbitals & A:ILE963 & Alkyl \\
\hline 4.703 & Pi-Alkyl & :RES1 & Pi-Orbitals & A:MET953 & Alkyl \\
\hline 5.287 & Pi-Alkyl & :RES1 & Pi-Orbitals & A:ILE963 & Alkyl \\
\hline
\end{tabular}

However, when the hydrogen bond contributions computed were compared to the $\mathrm{X} 6 \mathrm{~K}$ its energy was significant slightly lower $(-7.551 \mathrm{kcal} / \mathrm{mol})$ but comparable to that of PF-04691502. Furthermore, the hydrogen bond formed by INK with VAL882, ASP964 and GLY880 amino acids as shown in Table 6, were consistent with the interactions type found in the previously discussed inhibitors (PF-04691502, Palomid 529), suggesting these compounds may be acting with similar mechanism. nevertheless, INK formed other interactions that helped stabilze the ligand within the complex, such interactions are Pi-Pi Stacked, Pi-Alkyl and Alkyl-Alkyl hydrophobic interactions.

\section{Conclusions}

An mTOR kinase pdb file was collected from the RSCB site with a pdb identification number of 4JT6. The mTOR kinase was reported by numerous researchers as a viable target for a systematic inhibition of breast cancer cells. The molecular docking study of all the dataset compounds as possible inhibitors were reported in the work and the results suggests that of all the FDA approved cancer drugs collected, PF-04691502 best inhibited 4JT6 (mTOR kinase). The binding affinity of PF-04691502 was significantly higher than all the other compounds that were studied even though their interaction types and points suggest they undergo similar reaction mechanism with the target site. PF-04691502 and INK can be used as possible lead compound for further drug designs, since the enthalpic contributions of these ligands suggest that they are more stable compared to the referenced ligand (X6K).

\section{Acknowledgements}

We would like to thank the management, BAZE University Abuja, Nigeria and specifically our Pro-Chancellor Baba Ahmed for all his support throughout the course of this research. We thank you and appreciate your efforts sir.

\section{Authors' contributions}

DEA and RS designed the work, JNA and GCO collected the data, DEA and CM carried out the computational studies, RS and KLA analysed the result and finally DEA, SAA and CM wrote and edited the manuscript. All authors read and approved the final manuscript.

Funding

No funding was received.

Availability of data and materials

All data and material are available upon request.

\section{Declarations}

Ethics approval and consent to participate Not applicable.

\section{Consent for publication}

Not applicable.

\section{Competing interests}

No competing interests to declare. 


\section{Author details}

1 Department of Chemistry, Baze University, Abuja, Nigeria. ${ }^{2}$ Department of Microbiology, Federal University of Technology, Minna, Nigeria.

Received: 22 September 2020 Accepted: 8 March 2021

Published online: 20 March 2021

\section{References}

Abdullahi M et al (2020) Virtual molecular docking study of some novel carboxamide series as new anti-tubercular agents. Eur J Chem 11(1):30-36

Arthur DE, Abechi SE (2019) Corrosion inhibition studies of mild steel using Acalypha chamaedrifolia leaves extract in hydrochloric acid medium. SN Appl Sci 1(9):1089

Arthur DE, Uzairu A (2018) Molecular docking study and structure-based design of novel camptothecin analogues used as topoisomerase I inhibitor. J Chin Chem Soc 65(10):1160-1178

Arthur DE et al (2018) Structure-based optimization of tyrosine kinase inhibitors: a molecular docking study. Netw Model Anal Health Inform Bioinform 7(1):9

Arthur DE et al (2019) Molecular docking studies on the interaction of $\mathrm{NCl}$ anticancer analogues with human phosphatidylinositol 4, 5-bisphosphate 3-kinase catalytic subunit. J King Saud Univ Sci

Arthur DE et al (2020) Quantitative structure-activity relationship (QSAR) and design of novel ligands that demonstrate high potency and target selectivity as protein tyrosine phosphatase 1B (PTP 1B) inhibitors as an effective strategy used to model anti-diabetic agents. J Recept Signal Transduct 1-20

Aylett $\mathrm{CH}$ et al (2016) Architecture of human mTOR complex 1. Science 351(6268):48-52

Bernard M et al (2020) Autophagy drives fibroblast senescence through MTORC2 regulation. Autophagy 1-13

Calejman CM et al (2020) mTORC2-AKT signaling to ATP-citrate lyase drives brown adipogenesis and de novo lipogenesis. Nat Commun 11(1):1-16

Chen J, Long F (2018) mTOR signaling in skeletal development and disease. Bone Res 6(1):1-6

Choi S et al (2020) mTOR signaling intervention by Torin1 and XL388 in the insular cortex alleviates neuropathic pain. Neurosci Lett 718:134742

Choo AY, Blenis J (2009) Not all substrates are treated equally: Implications for mTOR, rapamycin-resistance, and cancer therapy. Cell Cycle 8(4):567-572

Davidson ER, Feller D (1986) Basis set selection for molecular calculations. Chem Rev 86(4):681-696

Davis NM et al (2014) Deregulation of the EGFR/PI3K/PTEN/Akt/mTORC1 pathway in breast cancer: possibilities for therapeutic intervention. Oncotarget 5(13):4603

Dienstmann $\mathrm{R}$ et al (2014) Picking the point of inhibition: a comparative review of PI3K/AKT/mTOR pathway inhibitors. Mol Cancer Ther 13(5):1021-1031

Gericke B et al (2020) Selective inhibition of mTORC1/2 or PI3K/mTORC1/2 signaling does not prevent or modify epilepsy in the intrahippocampal kainate mouse model. Neuropharmacology 162:107817

Hara K et al (2002) Raptor, a binding partner of target of rapamycin (TOR), mediates TOR action. Cell 110(2):177-189

Hehre WJ, Huang WW (1995) Chemistry with computation: an introduction to SPARTAN. Wavefunction, Inc
Jhanwar-Uniyal M et al (2019) Diverse signaling mechanisms of mTOR complexes: $\mathrm{mTORC} 1$ and $\mathrm{mTORC} 2$ in forming a formidable relationship. Adv Biol Regul 72:51-62

Keith CT, Schreiber SL (1995) PIK-related kinases: DNA repair, recombination, and cell cycle checkpoints. Science 270(5233):50-50

Kim D-H et al (2002) mTOR interacts with raptor to form a nutrient-sensitive complex that signals to the cell growth machinery. Cell 110(2):163-175

Knudsen JR et al (2020) Growth factor-dependent and-independent activation of mTORC2. Trends Endocrinol Metab 31(1):13-24

Laplante M, Sabatini DM (2012) mTOR signaling in growth control and disease. Cell 149(2):274-293

Li Z et al (2004) Personal experience with four kinds of chemical structure drawing software: review on ChemDraw, ChemWindow, ISIS/Draw, and ChemSketch. J Chem Inf Comput Sci 44(5):1886-1890

Liu Q et al (2011) Discovery of 9-(6-aminopyridin-3-yl)-1-(3-(trifluoromethyl) phenyl) benzo [h] $[1,6]$ naphthyridin-2 $(1 \mathrm{H})$-one (Torin2) as a potent, selective, and orally available mammalian target of rapamycin (mTOR) inhibitor for treatment of cancer. J Med Chem 54(5):1473-1480

Loewith R et al (2002) Two TOR complexes, only one of which is rapamycin sensitive, have distinct roles in cell growth control. Mol Cell 10(3):457-468

Matsuoka T, Yashiro M (2014) The role of PI3K/Akt/mTOR signaling in gastric carcinoma. Cancers 6(3):1441-1463

Meng D et al (2018) mTOR signaling in stem and progenitor cells. Development 145(1)

Neves MA et al (2012) Docking and scoring with ICM: the benchmarking results and strategies for improvement. J Comput Aided Mol Des 26(6):675-686

Paquette M et al (2018) mTOR pathways in cancer and autophagy. Cancers 10(1):18

Sarbassov DD et al (2004) Rictor, a novel binding partner of mTOR, defines a rapamycin-insensitive and raptor-independent pathway that regulates the cytoskeleton. Curr Biol 14(14):1296-1302

Schäfer A et al (1992) Fully optimized contracted Gaussian basis sets for atoms Li to Kr. J Chem Phys 97(4):2571-2577

Sciarretta S et al (2018) New insights into the role of mTOR signaling in the cardiovascular system. Circ Res 122(3):489-505

Shaw RJ, Cantley LC (2006) Ras, PI (3) K and mTOR signalling controls tumour cell growth. Nature 441(7092):424-430

Wander SA et al (2011) Next-generation mTOR inhibitors in clinical oncology: how pathway complexity informs therapeutic strategy. J Clin Investig 121(4):1231-1241

Wu ZR et al (2018) Inhibition of mTORC1 by IncRNA H19 via disrupting 4E-BP1/ Raptor interaction in pituitary tumours. Nat Commun 9(1):1-14

Yang $\mathrm{H}$ et al (2013) mTOR kinase structure, mechanism and regulation. Nature 497(7448):217-223

Zoncu R et al (2011) mTOR: from growth signal integration to cancer, diabetes and ageing. Nat Rev Mol Cell Biol 12(1):21-35

\section{Publisher's Note}

Springer Nature remains neutral with regard to jurisdictional claims in published maps and institutional affiliations.

\section{Submit your manuscript to a SpringerOpen ${ }^{\circ}$ journal and benefit from:}

- Convenient online submission

- Rigorous peer review

- Open access: articles freely available online

- High visibility within the field

Retaining the copyright to your article

Submit your next manuscript at springeropen.com 